\title{
Application of Queuing Systems with Ultimate Reliability to Optimize the Operation of Drones
}

\author{
Anna Kovalenko ${ }^{1}$ and Gennagi Rogachev ${ }^{1, *}$ \\ ${ }^{1}$ Samara state technical university, 443100, Samara, Russia
}

\begin{abstract}
Multi-criteria problem of optimization of maintenance rate for drones is set and solved. Analytical characteristics of a single-server queueing system with ultimate reliability and imitation models are applied. The analysis of economical and reliability characteristics dependence on some parameters is made.
\end{abstract}

\section{Introduction}

Drone is a complex object, result of solving the various technical and economic problems [1]. For sure, the primary problem is to develop the system of automatic drone control. In particular, the synthesis of control algorithms to stabilize flight trajectories is important [2]. However, like all complex technical devices with a large number of mechanical parts being a subject to constant deterioration, drones undergo break downs. One should also note the high cost, the responsibility for the quality of the problems solution and the effects of the environment [3]. Due to these reasons, the consideration of drone reliability in terms of mechanical failures and the solution of the drone survivability problem are quite relevant.

One of the methods of increasing the drones' reliability is to provide control at the design stage in the process of creating the devices [4] and to plan scheduled maintenance. In this paper, the optimization of maintenance rate of a drone operating according to the model of a single-server queueing system $[5,6]$ is made.

The main types of drone hardware failures are 1) Batteries discharge; 2) Engine failure; 3) Mechanical damage (improper motor shaft rotation, background noise and vibration, shear beam, etc.)

Preventive measures for the drone hardware include visual inspection to detect failures, maintenance (charging batteries, engine replacement or repair, shiftrays, etc). The essence of the strategy of drones maintenance described in [5] is the periodic (counting operating time) maintenance carried out after the end of task during which certain value of device operating time is reached.

\section{Problem setting}

Consider the operation of Geoksan 201, a drone of aircraft type. These drones are best suited for use over large areas of extended objects and shooting at a considerable distance. Geoskan 201 is capable of flying up to 180 minutes with a payload (camera), shooting up to 22 sq.km per one flight. However, due to the design features, it has to move constantly. It will not be able to hang in a necessary point or work in a confined space. Therefore, the device is effectively applied to shooting agricultural areas.

Suppose that the drone tasks arrive in a stream with the average inter-arrival time of 7 hours. The duration of task completion has a third-order Erlang distribution with the average of 5 hours (meaning that one task can include multiple drone flights). During a flight, a drone failure can occur. Then immediately the detection of the device, its transportation, failure analysis and restoration are carried out. The device failure is assumed to occur when the operating time reaches the random value having the Erlang fourth-order distribution with an average value of 300 hours. The operation time countdown starts at the moment of the first task income after restoration. The duration of restoration is a random variable with the average of 4 hours. Maintenance begins after the completion of the task during which device operation time has a predetermined level $\tau$. Both after maintenance and restoration reliability characteristics are completely renewed. The duration of maintenance is a random variable with the average of 1 hour. All the random variables are considered to be independent. The income per completed task is $C_{\text {ser }}=2000$ money units, the cost per time unit of restoration and maintenance are $c_{a}=1200$ money units/hour and $c_{p}=200$ money units/hour respectively.

\section{Optimization of the drone maintenance rate}

The following criteria, analytically defined in $[5,6]$, are taken into consideration: 
1) a positive criterion - average specific income $S(\tau)$ per unit of calendar time;

2) a negative criterion - average unit cost $C(\tau)$ per time unit of correct device operation;

3) a positive criterion - final probability $p^{0}(\tau)$ of a device standby mode;

4) a negative criterion - final probability $p^{-}(\tau)$ of maintenance or restoration; $p^{-}(\tau)$ also should not exceed a value $p^{-}$;

$$
\left\{\begin{array}{l}
S(\tau) \rightarrow \max _{\tau \in T}, \\
C(\tau) \rightarrow \min _{\tau \in T}, \\
p^{0}(\tau) \rightarrow \max _{\tau \in T}, \\
p^{-}(\tau) \rightarrow \min _{\tau \in T}, \\
T=\left\{\tau \mid p^{-}(\tau) \leq p^{-}\right\} .
\end{array}\right.
$$

If $p^{-}=0,05$, the restriction of the problem is $\tau \in T, T=\left\{\tau \mid p^{-}(\tau) \leq 0,02\right\}$. In our case, it means $\tau \in(0 ; \infty)$.

One can show that in this problem the set of Paretooptimal points can be obtained by maximizing the additive or multiplicative convolutions of partial criteria on the set $\mathrm{T}$ under different criteria weight coefficients.

Consider here an example of solving the optimization problem under equal criteria weight coefficients.

\section{Results of the optimization problem solution in Maple and Anylogic}

Without maintenance economical and reliability operation indexes are $S(\infty)=42,231$ money units/hour, $C(\infty)=9,639$ money units/hour, $p^{0}(\infty)=0,19522$, and $p^{-}(\infty)=0,00797$.

Under maintenance according to the strategy described in the problem setting, optimal values of specific criteria are $S\left(\tau_{\text {opt }}^{S}\right)=51,036$ money units/hour under $\tau_{\text {opt }}^{S}=81,395, \quad C\left(\tau_{\text {opt }}^{C}\right)=2,402$ money units/hour under $\tau_{\text {opt }}^{C}=81,066, \quad p^{0}\left(\tau_{\text {opt }}^{p 0}\right)=p^{0}(\infty)=0,19522$ under $\tau \rightarrow \infty$ and $p^{-}\left(\tau_{o p t}^{p-}\right)=0,00594$ under $\tau_{\text {opt }}^{p-}=206,306$.

Pareto set consists of the sets of specific criteria values corresponding the values of $\tau$ from semi-infinite interval $\Pi=\tau \in\left[\tau_{\text {opt }}^{C} ; \infty\right) \bigcup T=\left[\tau_{\text {opt }}^{C} ; \infty\right)$.

An additive convolution of specific criteria under equal weight coefficients $v_{s}=v_{c}=v_{p}=1 / 4$ reaches its maximum under $\tau_{o p t}^{V}=170,273$ hours; and $S\left(\tau_{\text {opt }}^{V}\right)=49,757$ money units/hour, $C\left(\tau_{\text {opt }}^{V}\right)=3,453$ money units/hour, $\quad p^{0}\left(\tau_{\text {opt }}^{V}\right)=0,19005$, $p^{-}\left(\tau_{o p t}^{V}\right)=0,00604$. At this point the values of specific criteria in absolute terms differ from the optima of specific criteria for $2,5 \%(S(\tau))$, for $43,7 \%(C(\tau))$, for

\section{$2,6 \%\left(p^{0}(\tau)\right)$ and for $1,7 \%\left(p^{-}(\tau)\right)$.}

The multiplicative convolution of specific criteria under equal weight coefficients $W_{s}=W_{c}=W_{p}=0,25$ reaches its minimum under $\tau_{o p t}^{W}=146,221$ hours; and $S\left(\tau_{o p t}^{W}\right)=50,262$ money units/hour, $C\left(\tau_{o p t}^{W}\right)=3,040$ money units/hour, $\quad p^{0}\left(\tau_{o p t}^{W}\right)=0,18958$, $p^{-}\left(\tau_{\text {opt }}^{V}\right)=0,00604$. At this point the values of specific criteria in absolute terms differ from the optima of specific criteria for $1,52 \%(S(\tau))$, for $26,52 \%(C(\tau))$, for $2,82 \%\left(p^{0}(\tau)\right)$ and for $5,75 \%\left(p^{-}(\tau)\right)$.

\section{The dependence of the optimal maintenance rate on some parameters of device}

Fig. 1, 2 represent graphs of economical characteristics against $\tau$ under different values of $c_{p}$.

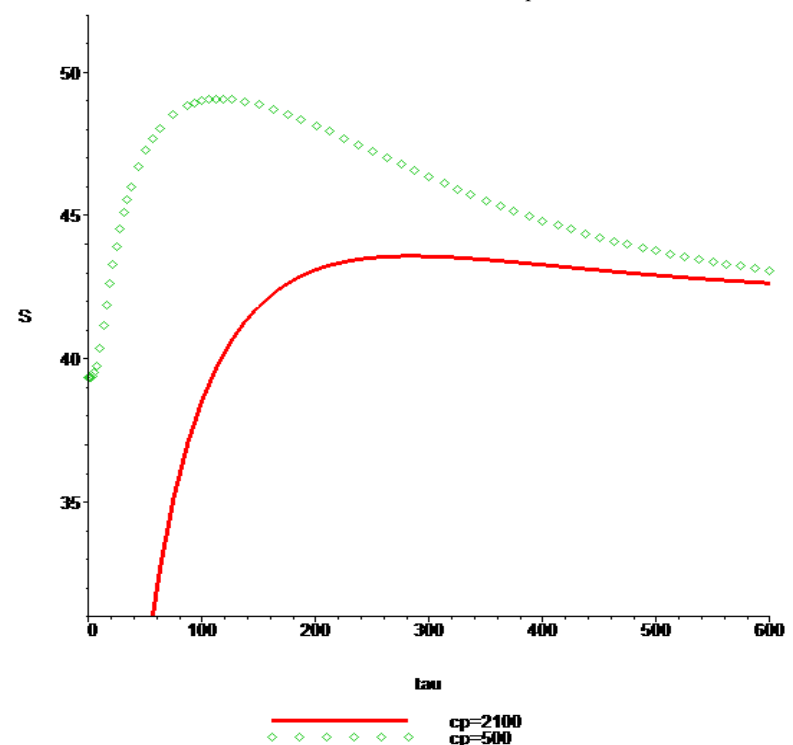

Fig.1 Graph of average specific income $S(\tau)$ against $\tau$ under different values of $c_{p}$.

With the increase of the cost $c_{p}$ per maintenance time unit:

- the optimal maintenance rate increases; it is more evident for specific economical criteria and less evident for multi-criteria optimization;

- the value of the specific average income per time unit decreases;

- the value of the specific average cost per time unit of correct device operation increases;

- the optimum of specific criteria becomes less evident (for the value of approximately $c_{p}=3000$ the cost function becomes monotonous, for $c_{p}=3650$ the income function does). 


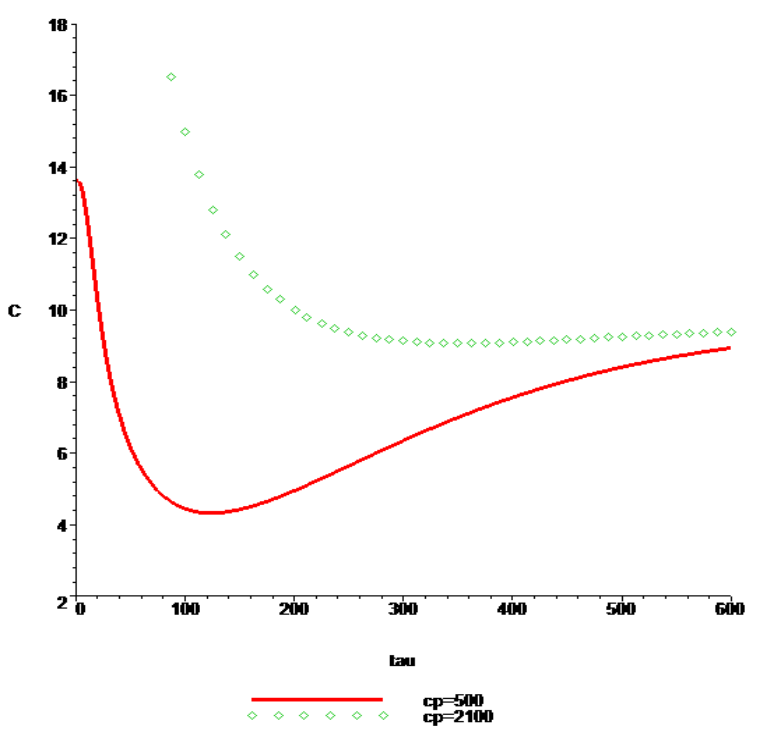

Fig.2 Graphs of average specific income $S(\tau)$ and average specific cost $C(\tau)$ against $\tau$ under different values of $c_{p}$.

With the increase of the intensity of income stream $\lambda$ :

- the optimal maintenance rate does not change significantly;

- the value of the specific average income per time unit increases;

- the value of the specific average cost per time unit of correct device operation increases;

- the optima of specific economical criteria become more evident, while the optimum of final probability of device maintenance or restoration becomes less evident (for $\lambda=0,33$ this criterion reaches its minimum under $\tau \rightarrow \infty)$.

\section{Simulation in Anylogic}

Discrete event simulation of the device operation is carried out with Anylogic software. The operation of this model is shown in Fig. 3. In the upper left corner there are model parameters. To the right of the box model, you can see the current results of the calculations. With this model it is possible to analyze the stationary, nonstationary characteristics of the system, to carry out optimization experiments and make statistical analysis and visualization (as in Fig. 4).

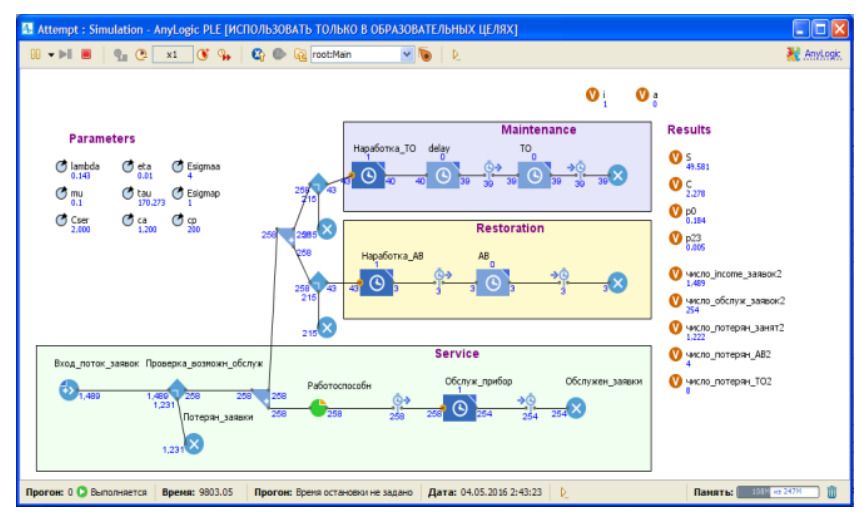

Fig. 3. Simulation process of drone operation.

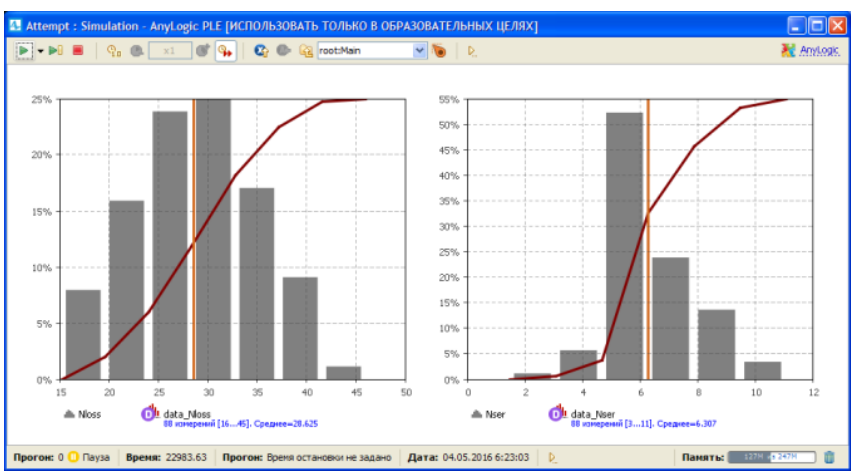

Fig. 4. Histograms of the numbers of lost and completed tasks (correspondingly) per regeneration period.

The optimization experiment in Anylogic can give us the values of optimal device rate for most of the criteria. However, you need 50 iterations with 20 replications (for the specific average income criterion) and much more for the other criteria and multiplicative convolutions to get results, coinciding with those calculated according to the analytical formulas up to 0,03 .

\section{Conclusions}

The high cost of drones, their accessories, and restoration, as well as the impossibility of direct inspection while their operation, results in the need for maintenance to improve hardware reliability. Carrying out drone maintenance according to the strategy proposed increases the average specific income per time unit for at least $15 \%$ and reduces the average specific cost per time unit of correct device operation more than 1,5 times. The final probability of device standby mode is reduced not more than for $2,7 \%$, and the final probability of maintenance or restoration slightly decreases.

This work was financially supported by Ministry of Education and Science of Russian Federation (basic part of government contract).

\section{References}

1. A. N. Katulev, Operation Research: Principles of Decision Making and Safety Ensurance (Tver', 1999)

2. A. A. Antanevich, Yu. F. Ikuas, L. A. Lobaty, Science and Technics, 5 (2010)

3. A. V. Betskov, I. V. Prokop'ev, Reliability and Quality of Complex Systems, 2(6) (2014)

4. I. I. Ogoltsov, N. B. Rozhnin, V. V. Sheval', Izvestiya TulSU. Technical Sciences, 1 (2012)

5. A.I. Peschansky, A.I. Kovalenko, Automatic Control and Computer Sciences, 50(6) (2016)

6. A. Kovalenko, G. Rogachev, ITM Web of Conferences, 6 (2016) 